\title{
Laboreal
}

Volume $5 \mathrm{~N}^{\circ} 2$ | 2009

Varia

\section{Desarrollo de la seguridad y actividades mediatizantes : el caso del trabajo en la construcción}

Desenvolvimento da segurança e actividades mediadoras : o caso do trabalho de construção de estruturas em estaleiros de construção civil

Développement de la sécurité et activités médiatisantes : le cas du travail de gros-œuvre sur les chantiers de bâtiment

Safety development and mediating activities : the case of carcassing work in construction sites

\section{Julie Duboscq}

\section{OpenEdition}

\section{Journals}

Edición electrónica

URL: http://journals.openedition.org/laboreal/9885

DOI: 10.4000/laboreal.9885

ISSN: 1646-5237

Editor

Universidade do Porto

Referencia electrónica

Julie Duboscq, « Desarrollo de la seguridad y actividades mediatizantes : el caso del trabajo en la construcción », Laboreal [En línea], Volume 5 N² | 2009, Publicado el 01 diciembre 2009, consultado el 24 septiembre 2020. URL : http://journals.openedition.org/laboreal/9885 ; DOI : https://doi.org/ 10.4000/laboreal.9885

Este documento fue generado automáticamente el 24 septiembre 2020

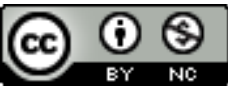

Laboreal está licenciado com uma Licença Creative Commons - Atribuição-NãoComercial 4.0 Internacional. 


\section{Desarrollo de la seguridad y actividades mediatizantes : el caso del trabajo en la construcción}

Desenvolvimento da segurança e actividades mediadoras : o caso do trabalho de construção de estruturas em estaleiros de construção civil

Développement de la sécurité et activités médiatisantes : le cas du travail de gros-œuvre sur les chantiers de bâtiment

Safety development and mediating activities : the case of carcassing work in construction sites

Julie Duboscq

\section{REFERENCIA}

Duboscq, J. (2009). Développement de la sécurité et activités médiatisantes : le cas du travail de gros-oeuvre sur les chantiers de bâtiment. Thèse de doctorat en psychologie, CNAM, Paris.

NOTA DEL EDITOR

Manuscrito recibido en : Junio /2009

Aceptado tras peritage en : septiembre/2009 


\section{Problemática : Mejorar la seguridad en las obras de construcción}

1 Una constructora de tamaño mundial solicitó el equipo clínica de la actividad en 2004 para buscar nuevas formas de pensamiento y de acción con el fin de reducir el número de accidentes en obras de la construcción y más generalmente, para mejorar la seguridad del trabajo. El grupo teme de que aumente la tasa de frecuencia de accidentes [1] estancado en alrededor de 25 accidentes por cada millón de horas trabajadas desde la década de 2000. El reto para la investigación también es sectorial. De hecho, en Francia [ $\left.{ }^{2}\right]$ y a nivel internacional [3], el ámbito de la Construcción y Obras Públicas aún soporta una tasa de frecuencia de accidentes significativamente mayor en comparación con otros sectores.

2 En esta empresa, las acciones preventivas se refieren principalmente a la renovación de equipos, materiales, equipos de seguridad y métodos de trabajo asociados. Estas acciones resultan insuficientes para continuar la disminución de la tasa de frecuencia. Del mismo modo, los incentivos en términos de primas y concursos relacionados con la seguridad no proporcionan una mejora significativa y una seguridad duradera. A finales de 2004, las direcciones de Investigación y Prevención del grupo desean profundizar en las dimensiones "humanas" del trabajo, solicitando 4 investigadores de psicología del trabajo y de ciencias de la gestión [4]. Ya en 2006, estas direcciones instauran un procedimiento semanal de diálogo corto sobre la seguridad de los equipos de terreno (trabajadores, jefes, conductor de obra) llamado "cuarto de hora de seguridad". Este diálogo inter-oficio tampoco consigue transformar a fondo las actitudes de los trabajadores. Los trabajadores asisten con comedimiento. Los jefes y los directivos tienden a repetir las normas de seguridad, sin debate real con los trabajadores y entre los trabajadores sobre los problemas surgidos en la actividad.

3 Ante esta problemática, optamos por estudiar las condiciones y los mecanismos de desarrollo de la seguridad en las obras de construcción, mediante el desarrollo de la actividad en sí misma de los actores de la obra, de acuerdo con el enfoque históricocultural de análisis de la actividad de Vygotski (1978) y algunos de sus mas recientes desarrollos (Clot, 2008).

\section{Marco teórico : análisis y desarrollo de la actividad ordinaria}

4 Nuestra trayectoria teórica nos permitió distinguir varias perspectivas entre los enfoques de la seguridad. En primer lugar, hemos caracterizado el primer enfoque, principalmente cognitivo, enjuiciando la violación de la seguridad no solamente a través del elemento técnico, sino también los errores humanos (Reason, 1993; Rasmussen, Pejtersen \& Goodstein, 1994) y las disfunciones de la organización (Llory , 2003). Este enfoque se extiende al estudio de la fiabilidad de la organización (Bourrier, 1999) dentro de un sistema más amplio (Leplat \& De Terssac, 1990), arriesgando limitar la importancia de las situaciones y de las propias actividades.

5 Escogimos entonces otra perspectiva en la que la seguridad es el resultado de "la acción situada" de sujetos en sus interacciones con los demás y su entorno. La planificación del trabajo, considerada como un recurso de la actividad no es suficiente (Suchman, 1987). 
Sin embargo, el sistema de cognición que distribuye la carga cognitiva entre los actores de la situación y sus instrumentos garantiza alta seguridad (Hutchins, 1994). Este enfoque tiene, sin embargo, un límite. Centrándose en la situación, no enjuicia la actividad en su plan histórico y subjetivo, lo que, en nuestra opinión, oscurece algunos resortes del desarrollo de la actividad en sí y por lo tanto de la seguridad.

Nuestro trabajo se refiere a un tercer enfoque para la seguridad, que analiza la actividad con las actores de la misma y actualiza su potencial de desarrollo. Este enfoque esclarece, entre otras cosas, la utilización de "saberes de precaución" (Cru, 1995) de los trabajadores, posible respuesta a la "prescripción difusa" del trabajo (Duc, 2002), en "justo a tiempo" (Gaudart, Delgoulet \& Chassaing, 2008), que rara vez implica a los trabajadores (Six, 2003). Procedente de la perspectiva histórico-cultural de la actividad (Vygotski, 1978), este análisis de la actividad completa la actividad realizada y visible por otras vías realizables o no en la situación, a veces incluso en conflicto. Para el autor, la actividad humana no se ve sino durante el desarrollo (ibid., p. 64). De hecho, "el comportamiento realizado sólo es una pequeña fracción de lo que es posible" (Vygotski, 2003, p. 74). Según esta tradición, el desarrollo de la actividad, en sus logros en el lenguaje y la práctica, permite vislumbrar la actividad "real" (Clot, 1999) y observar los mecanismos del desarrollo de la actividad y por lo tanto de la seguridad.

\section{Metodología y método de intervención}

Tratamos de observar el desarrollo de actividades de construcción más seguras [5]. Para ello, llevamos a cabo una intervención en una obra por un período de 20 meses, buscando desarrollar la actividad de los profesionales.

En primer lugar, con los comanditarios, hemos identificado dos sitios de intervención de acuerdo a los criterios de significatividad [ $\left.{ }^{6}\right]$ y viabilidad [ $\left.{ }^{7}\right]$ : la extensión de un colegio, obra de dimensiones clásicas (1 grúa, 20 trabajadores, 11 meses de trabajo) y la construcción de una torre con importantes recursos (3 grúas, hasta 150 trabajadores, 28 meses de trabajo), con una plantilla de directivos y gerentes de construcción suficientes para realizar un análisis colectivo de su actividad. Entonces, siguiendo el método de autoconfrontaciones indirectas (Clot, Faïta, Fernández \& Scheller, 2001), hemos combinado 3 colectivos (15 trabajadores, 6 jefes y 6 directivos) para llevar a cabo un coanálisis de su actividad de preparación y ejecución de la obra. El método se ha desarrollado en varias etapas : observar varios cientos de horas el trabajo en la obra y crear tres colectivos de análisis, filmar 6 profesionales de cada grupo en algunas situaciones de trabajo ordinario, comparables entre pares $y$, a veces entre colectivos, $y$ seguidamente analizarlas en autoconfrontation directa e indirecta y luego con el colectivo plenario. Hemos analizado en profundidad 5 situaciones perfilando la actividad habitual de los trabajadores, incluso la de los jefes y ejecutivos. Para ello, hemos realizado ciertos ajustes de método (por ejemplo, limitar la duración de la entrevista de los directivos y jefes y hacerlas en varias veces). Sin embargo, hemos mantenido 3 principios metodológicos que creemos son esenciales en la clínica de la actividad: la inversión de los protagonistas de la observación, la alternancia de las actividades de análisis y el trabajo habitual y la ampliación progresiva del diálogo sobre la actividad, de lo individual a lo colectivo. 


\section{Resultados y análisis}

9 Después de la intervención, no todos los desarrollos de la actividad de los participantes contribuyeron a mejorar la seguridad. Por "desarrollo" nos referimos a cualquier actividad de mediatizante (Clot, 2008) "creadora de objetos, instrumentos y destinatarios" (p. 21). Entre los desarrollos iniciados, hemos diferenciado desarrollos efectivos, impedidos y ficticios para la seguridad. Los desarrollos efectivos corresponden a la adopción por los obreros de modos operatorios más seguros y la reconcepción de tareas. Por ejemplo, los obreros enganchan las eslingas de la grúa a los paneles prefabricados utilizando una escalera sujetada en un rack de almacenamiento y no una escalera móvil posada en los paneles. Los desarrollos se ven impedidos cuando están parados en una fase inicial, prohibiendo cualquier mejora de la seguridad. Por último, los desarrollos ficticios corresponden a la prestación de instalaciones más seguras que los trabajadores no utilizan. Este es el caso de los arneses que los obreros no enganchan a las cuerdas de salvamento. Por lo tanto, la seguridad no mejora sin el desarrollo de la propia actividad en determinadas condiciones y según ciertos procesos.

\subsection{Condiciones de desarrollo de una actividad más segura}

Estudiando el desarrollo real observado en una situación, identificamos 10 condiciones para el desarrollo de una actividad más segura :

11 Condiciones relacionadas con la actividad práctica $\left.{ }^{8}\right]$ analizada

1. El sujeto considera la actividad práctica como problemática

2. Se analiza la actividad en todas sus dimensiones

3. La actividad se analiza en relación con realizaciones concretas por el sujeto

12 Condiciones relacionadas con los participantes

1. 4. El sujeto de la actividad participa al análisis

2.5. Otro profesional, con oficio similar al sujeto, participa al análisis

3. 6. Este otro profesional también participa en el análisis de una actividad propia

4. 7. Los participantes son de mismo nivel jerárquico

5. 8. Los participantes tienen una experiencia significativa

13 Condiciones relacionadas con los problemas no solucionados por el análisis

1. 9. Identificación de personas apropiadas, competentes para ayudar a resolverlos

2. 10. Transmisión efectiva y objectiva de los problemas no resueltos, a estas personas

14 Luego, se identifican 12 secuencias suficientemente documentadas entre todos los desarrollos que surgen en 5 situaciones, para poner a prueba las condiciones mencionadas. Todas estas condiciones son satisfechas en la secuencia de desarrollo de la actividad de eslingado del obrero C. Por el contrario, le falta al menos una de las 10 condiciones en las 6 secuencias de desarrollo impedido como en las 5 secuencias de desarrollo ficticio de la seguridad.

\subsection{Proceso de desarrollo de una actividad más segura}

En la secuencia que comprende las 10 condiciones, nos fijamos en el proceso por el cual la actividad de $\mathrm{C}$ es realmente más segura. Esta secuencia se extiende desde la 
realización de una autoconfrontación indirecta entre $\mathrm{C}$ y otro trabajador, hasta la adopción posterior por $\mathrm{C}$ de un nuevo modo de eslingado de los paneles prefabricados, considerado más seguro. Analizamos los procesos por los cuales la actividad de C se desarrolla durante la autoconfrontación indirecta y a continuación en la actividad práctica.

Por un lado, de acuerdo con algunos modelos bajtinianos de análisis del discurso, la actividad de lenguaje de los protagonistas durante la autoconfrontación indirecta se desarrolla transformando sucesivamente el objeto del discurso (Sitri, 2003) a través de varias repeticiones (Bernicot, Salazar-Orvig \& Veneziano, 2006 ; Vion, 2006) de palabras para sí mismo (autorepetición) y palabras de otros (hétérorepeticiones) más o menos completas. El desarrollo del lenguaje también se caracteriza por la presencia simultánea o secuencial de varias direcciones del discurso, a los pares, a si mismo y a un sobredestinatario (Salazar-Orvig \& Grossen, 2004 ; Peytard, 1996). Estos movimientos de objetos y direcciones son recursos para el desarrollo del análisis.

Por otra parte, basándose en el trabajo de Vygotski (2003) y Wallon (1970), hacemos la hipótesis de que ciertos objetos y herramientas del análisis desarrollados en autoconfrontación indirecta, se convirtieron, por migración funcional en instrumentos del desarrollo de una actividad práctica más segura.

\section{Perspectivas}

Cabe preguntarse si las 10 condiciones, suficientes para engendrar el desarrollo de una actividad más segura, son todas necesarias. Esta cuestión nace en un contexto donde la organización se muestra reacia a tomar plenamente en cuenta las condiciones. De hecho estas condiciones establecen un foro específico para el diálogo entre pares y entre agentes con funciones diferentes o de niveles jerárquicos diferentes. Este foro representa un coste para la empresa relacionado con el tiempo : tiempo "improductivo" y riesgo de desorganización o retrasos en las obras de construcción. Además, el análisis de los conflictos de la actividad en su complejidad se opone a una cultura de resolución sencilla e inmediata de los problemas. Además, el establecimiento y la conservación de las condiciones necesitan un apoyo externo, añadiendo un coste a los costes estimados. El apoyo de los dirigentes en el proceso y su participación es indispensable para iniciar estas inversiones.

\section{BIBLIOGRAFÍA}

Bourrier, M. (1999). Le nucléaire à l'épreuve de l'organisation. Paris : PUF.

Bernicot, J., Salazar Orvig, A. \& Veneziano E. (2006). Les reprises : dialogue, formes, fonctions et ontogenèse. La linguistique, 42, 29-50.

Clot, Y. (1999). La fonction psychologique du travail. Paris : PUF. 
Clot, Y. (2008). Travail et pouvoir d'agir. Paris : PUF.

Clot, Y., Faïta, D., Fernandez, G. \& Scheller, L. (2001). Entretiens en autoconfrontation croisée : une méthode en clinique de l'activité. Revue Education Permanente, 146, 17-25.

Cru, D. (1995). Règles de métier, langue de métier : dimension symbolique au travail et démarche participative de prévention. Le cas du bâtiment et des travaux publics, Mémoire pour l'obtention du diplôme de l'Ecole Pratique des Hautes Etudes, 126p.

Duc, M. (2002). Le travail en chantier. Paris : Octarès.

Gaudart, C., Delgoulet, C. \& Chassaing, K. (2008). La fidélisation de nouveaux dans une entreprise du BTP : Approche ergonomique des enjeux et des déterminants. Activités, 5(2), 2-24, http:// www.activites.org/v5n2/v5n2.pdf

Hutchins, E. (1994). Comment le cockpit se souvient de ses vitesses. Sociologie du Travail, 4, 110-117.

Leplat, J. \& De Terssac, G. (Eds) (1990). Les facteurs humains de la fiabilité dans les systèmes complexes. Toulouse : Octarès Editions.

Llory, M. (2003). L'appréhension du risque, entre formalisme et intuition. In F. Hubault (eds), Travailler, une experience quotidienne du risque? (pp. 131-146). Toulouse : Octarès Editions.

Peytard, J. (1996). Discours intérieur vs discours rapporté chez Volochinov/Bakhtine. Cahiers du français contemporain, 3, 9-26. Tunis : Publication du Credif.

Rasmussen, J., Pejtersen, A.M. \& Goodstein, L.P. (1994). Cognitive systems engineering. New York: J. Wiley.

Reason, J. (1993). L'erreur humaine. Paris: PUF.

Salazar-Orvig, A. \& Grossen, M. (2004). Représentations sociales et analyse de discours produit dans des focus groups : un point de vue dialogique. Bulletin de Psychologie, tome 57, $n^{\circ} 3$, 263-272.

Sitri, F. (2003). L'objet du débat. Paris : Presses Sorbonne Nouvelle.

Six, F. (2003). Préparation du travail et gestion des risques dans l'activité. In F. Hubault (eds), Travailler, une expérience quotidienne du risque. Toulouse : Octarès Editions.

Suchman, L. A. (1987). Plans and situated actions: the problem of human-machine communications. Cambridge: Cambridge University Press.

Vion, R. (2006). Reprises et modes d'implication énonciative. La linguistique, 42, 11-25. Paris : Presses Universitaires de France.

Vygotski, L. (1978). Mind in society: The Development of Higher Psychological Processes. Cambridge: Harvard University Press.

Vygotski, L. (1925-1932/2003). Conscience, inconscient, émotions. Paris : La Dispute.

Wallon, H. (1970). De l'acte à la pensée. Paris : Flammarion.

\section{NOTAS}

1. La tasa de frecuencia es la proporción entre el número de accidentes notificados con baja de hasta más de un día y el número de millones de horas trabajadas. 
2. En Francia, en 2006, la tasa de incidencia de accidentes en la construcción fue del 53,4 en comparación con las tasas de incidencia no superior a 34 en otras industrias y servicios (fuente: Instituto Nacional de Investigación y Seguridad).

3. Estudios de la EASHW (Agencia Europea para la Seguridad y la Salud en el Trabajo).

4. Se dedican a la investigación, dos investigadores en clínica de la actividad (el autor de esta tesis y su director, Yves Clot) y otros dos investigadores en gestión (Benoît Tricard, doctorante, y su director, Philippe Lorino).

5. Por actividad más segura entendemos una actividad con mayor seguridad para los interesados de esta actividad con relación a la situación anterior. Los profesionales valoran ellos mismos esta seguridad.

6. Por "significatividad" nos referimos a la posibilidad de dar cuenta de los problemas de seguridad relacionados con la actividad de trabajo regular. Estos problemas pueden encontrarse en muchas obras.

7. Por «viabilidad», nosotros entendemos la posibilidad de conducir un co-análisis con los profesionales.

8. La actividad práctica es la actividad ordinaria de trabajo objeto del análisis.

\section{AUTOR}

\section{JULIE DUBOSCQ}

Centre de Recherche “Travail et Développement”, Equipe de Clinique de l'Activité, 41, rue GayLussac, 75005 Paris, France.

jduboscq@hotmail.com 